\title{
Physical activity in older people: a systematic review
}

Fei Sun ${ }^{1}$, lan J Norman ${ }^{2}$ and Alison E While $2^{2^{*}}$

\begin{abstract}
Background: Physical activity (PA) in older people is critically important in the prevention of disease, maintenance of independence and improvement of quality of life. Little is known about the physical activity of the older adults or their compliance with current physical activity guidelines.

Methods: A systematic literature search of the published literature was conducted. Included were published reports of original research that independently reported: the PA level of non-institutional older adults (aged 60 years and over); and the proportion of older adults in the different samples who met PA recommendations or guidelines. The review was restricted to studies published since 2000 to provide a current picture of older adults' PA levels.
\end{abstract}

Results: Fifty three papers were included in the review. The percentage of older adults meeting recommended physical activity ranged from $2.4-83.0 \%$ across the studies. Definitions of "recommended" physical activity in older adults varied across the studies as did approaches to measurement which posed methodological challenges to data analysis. Older age groups were less likely than the reference group to be regularly active, and women were less likely than men to achieve regular physical activity, especially leisure time physical activity, when measured by both subjective and objective criteria.

Conclusion: The review highlights the need for studies which recruit representative random samples of community based older people and employ validated measurement methods consistently to enable comparison of PA levels over time and between countries.

Keywords: Physical activity, Exercise, Older people, Older adults

\section{Background}

Regular physical activity (PA) can bring significant health benefits to people of all ages and the need for PA does not end in later life with evidence increasingly indicating that PA can extend years of active independent living, reduce disability and improve the quality of life for older people [1]. Indeed a large scale longitudinal 8 year study found that every additional 15 minutes of daily PA up to 100 minutes per day resulted in a further $4 \%$ decrease in mortality from any cause [2]. Increasing PA will help minimise the burden on health and social care through enabling healthy ageing $[3,4]$.

There is no known review of PA among older people and it is not known whether active older people comply with recommended PA levels. Understanding the extent

\footnotetext{
* Correspondence: alison.while@kcl.ac.uk

${ }^{2}$ King's College London, Florence Nightingale School of Nursing \& Midwifery, 57 Waterloo Road, London SE1 8WA, UK

Full list of author information is available at the end of the article
}

of PA will provide a global perspective of PA among older people within the context of an increasing desire to promote PA goals across all age groups. The aim of this review was to establish global levels of PA among older people as reported in the published literature. Establishing PA prevalence in older community dwelling people provides a baseline against which changes in PA can be measured, international comparisons drawn and the success or otherwise of public health interventions to increase PA evaluated.

\section{Methods}

\section{Search strategy}

The following methods were used to locate relevant published studies from January 2000 - 11 April 2011. Electronic searches of computerized databases were carried out on English language databases (The Cochrane Library, PubMed, MEDLINE, EMBASE, CINAHL, PsycINFO, 
British Nursing Index (BNI) and Scopus) and Chinese databases: Chinese Biomedical, VIP Chinese Science Journals and WANFANG DATA. Keyword combinations for electronic database searches are listed in Table 1. The search was limited to the English and Chinese languages.

\section{Selection criteria}

Papers were reviewed if they met the following criteria: (1) original research; (2) independently reported the PA level of non-institutional older people (adults aged 60 years and over); (3) reported the proportion of any of PA recommendation or guidelines achieved by the sample.

No attempt was made to access unpublished studies or other 'grey' literature. The study selection process is set out in Figure 1.

\section{Data extraction and appraisal}

All authors devised the research strategy. Initial screening was undertaken by one researcher (FS) and then checked by another (AEW). Disagreements about inclusion were discussed until agreement was reached. One researcher (FS) extracted the following data from the selected studies: country of study, study sample, measurements, recommendations and main results. Another researcher (AEW) verified the extracted data and made corrections where necessary. Disagreements were resolved by reference to the third researcher (IJN). All three researchers contributed to the synthesis of the data.

\section{PA recommendations or guidelines}

Definitions of "recommended" PA in older adults varied across the studies with some studies employing two or more guidelines. These guidelines are summarized in Table 2. The minimum recommended PA level in the guidelines in most studies was 150 minutes of moderate or vigorous PA per week and we adopted this standard as the desired PA level for the purpose of this review. We categorised the selected studies according to their underpinning PA guidelines, and identified studies as falling into one of two groups according to the detail provided by the study authors on the guidelines followed. Twenty six studies which stated only the total amount of PA per week were classified within the 'less detailed guideline group'. Studies which stated the weekly frequency and daily dosage towards the total amount of weekly PA were classified as being in the 'detailed guideline group'. According to the Physical Activity Guideline Advisory Committee's report [5], detailed guidelines, such as 1995 CDC/ACSM guideline is too specific. In other words, the existing scientific evidence cannot distinguish the health benefits of $30 \mathrm{mi}-$ nutes of PA on 5 days a week from those gained through 50 minutes of PA on 3 days a week.

\section{Results and discussion}

\section{An overview of selected papers}

A total of 53 papers met the inclusion criteria. The main findings of the selected studies are reported in Tables 3 and 4.

Most of the studies were conducted in the United States and Australia (USA $n=19$; Australia $n=10$; UK $\mathrm{n}=5$; Brazil $\mathrm{n}=4$; China $\mathrm{n}=4$; Canada $\mathrm{n}=4$; New Zealand $\mathrm{n}=1$; Colombia $\mathrm{n}=1$; South Africa $\mathrm{n}=1$; Greece and Cyprus $n=1$; Cyprus $n=1$; Sweden $n=1$; Switzerland $n=1$ ). Forty nine papers reported cross-sectional studies and four reported longitudinal studies. The sample sizes ranged from 54 - 43,259. The number of studies conducted each year over the search period was not constant (1990-1994, $\mathrm{n}=2 ; 1995-1999, \mathrm{n}=12 ; 2000-2004$, $\mathrm{n}=32 ; 2005-2009, \mathrm{n}=18 ; 2010-2011, \mathrm{n}=2$ ) with more than half being conducted between 2000 and 2004.

Forty seven studies measured PA intensity, duration, and frequency using subjective measures (interview or self-reported questionnaires) and six reported objective data gathered using an accelerometer. Two studies compared subjective and objective data measurements. While 39 studies recorded PA taken in periods of $10 \mathrm{mi}$ nutes or more, other studies recorded all PA.

Physical activity comprises leisure-time PA, occupational PA, household PA and transportation PA. Leisuretime PA (LTPA) was most often measured and compared to the criterion for meeting PA recommendations. However, occupational, household and transportation PA were gathered in some studies. PA volume was calculated differently across the studies including: total metabolic equivalents (METs), minutes of weekly PA; minutes of participation in and frequency of PA during the week; kilocalories expended per kilogram of weight per day; and time in moderate to vigorous PA from accelerometers. To calculate minutes of weekly moderate to vigorous PA, some authors summed the duration of moderate and vigorous PA (MPA+VPA) while others doubled the time of vigorous PA because of its higher

Table 1 Search terms

\begin{tabular}{ll}
\hline Facets & Search terms \\
\hline PA & Exercise/s; physical activity/activities \\
Old people & Aged, old people, elderly, elders, aging adult, ageing adult, old men, old women, older people, older men, older women, older person \\
Research & Prevalence, health survey/surveys, survey/surveys, surveillance, statistics, epidemiologic \\
\hline
\end{tabular}






intensity (MPA+2VPA). The definition of moderate and vigorous $\mathrm{PA}$ also varied across the studies. For instance, the minimum cut off of moderate PA varied from 3 MET, 3.3 MET to 4 MET across the studies. Given the variety of methods of data collection and calculation of PA level, a meta-analysis was not attempted.
Levels of PA within recommendations or guidelines

Thirty two studies adopted the more detailed PA guidelines while 26 studies were in the less detailed group with some studies employing two or more guidelines. Although most of the PA guidelines stated the total PA amount which should be accumulated in bouts of at 
Table 2 Recommended levels within PA guidelines used in the selected studies

\begin{tabular}{|c|c|c|c|c|c|}
\hline \multirow{2}{*}{\multicolumn{2}{|c|}{$\begin{array}{l}\text { PA type /if accumulated by } 10 \\
\text { min bouts Guidelines }\end{array}$}} & \multicolumn{2}{|c|}{ Self-reported PA studies } & \multirow{2}{*}{\multicolumn{2}{|c|}{$\frac{\text { Objectively measured PA studies }}{10 \text { minute bouts }}$}} \\
\hline & & \multicolumn{2}{|c|}{10 minute bouts } & & \\
\hline & & $Y^{9}$ & $\mathrm{~N}^{10}$ & $Y^{9}$ & $N^{10}$ \\
\hline \multirow[t]{3}{*}{ More detailed } & CDC-ACSM $1995^{1}$ & $3,8,26,31,33$ & $1^{*}, 5^{*}, 6^{*}, 10,17,24,30,46$ & $50,52,53$ & 46 \\
\hline & HP $2010^{2}$ & $13,14,16,19,28,32,39,41,42,43,47,48$ & $11,22,49$ & & \\
\hline & $\mathrm{CAPG}^{3}$ & 35 & & & \\
\hline \multirow[t]{5}{*}{ Less detailed } & $3 K_{K D}^{4}$ & 29,35 & & & \\
\hline & $500 \mathrm{METs}^{5}$ & 36,38 & & 38 & \\
\hline & SGR $1996^{6}$ & $3,4 *$ & 15 & & \\
\hline & PAGA2008 ${ }^{7}$ & $18,23,20,21,27,38,40,43,44,45$ & & $38,51,52,53$ & \\
\hline & 150 mins/week ${ }^{8}$ & $2,7^{*}, 12,9^{*}, 34^{*}, 37^{*}$ & 25 & & \\
\hline
\end{tabular}

${ }^{1}$ CDC-ACSM 1995: Centers for Disease Control and Prevention and the American College of Sports Medicine 1995 PA Recommendation states that “Every U.S. adult should accumulate 30 minutes or more of moderate-intensity PA on most, preferably all, days of the week". The cut point of $\geq 150$ min, and 5 times weekly, was treated as the same criterion.

${ }^{2}$ HP 2010: Healthy people 2010, recommended levels of activity are 30 minutes of moderate activity on at least five days a week or 20 minutes or vigorous activity three times a week.

${ }^{3}$ CPAG recommendation: It recommends $60 \mathrm{~min}$ of light activity daily, 30 to $60 \mathrm{~min}$ of moderate activity 4 days a week, or 20 to 30 min of vigorous activity 4 days a week. We choose the combination of moderate and vigorous PA.

${ }^{4}$ 3.0 KKD: Canada National PA goal (Canadian Fitness and Lifestyle Research Institute 2005): Energy expenditure >1.5 KKD has been used to categorize those who are at least moderately active and who, thus, meet national physical activity goals. We adopted 3.0 KKD (active threshold, 1.5 KKD is moderate active threshold) so that it is comparable to the other guidelines. (KKD, kilocalories per kilogram per day).

500 METs: modified SGR that only includes the energy expenditure component, i.e., 500 METs per week.

${ }^{6}$ SGR 1996: modified from US Surgeon General's Report recommendation, demands a weekly amount of PA as approximately $1000 \mathrm{kcal} \cdot \mathrm{wk}^{-1}$ (or $150 \mathrm{kcal} / \mathrm{day}$ ).

7 PAGA 2008 (2008 PA Guidelines for Americans): adults should participate weekly in at least 150 minutes of moderate intensity aerobic activity, 75 minutes of vigorous-intensity aerobic activity, or an equivalent combination. This is the same criterion used in the recommendation from WHO, UK, and Canada's Physical Activity Guide for Healthy Active Living.

${ }^{8} 150$ minutes/week: modified PAGA 2008 that only includes 150 minutes PA per week.

$Y^{9} P A$ amount was accumulated by 10 minute bouts.

$\mathrm{N}^{10} \mathrm{PA}$ amount was not accumulated by 10 minute bouts.

* Studies in which only walking component in PA was accumulated by 10 minute bouts.

*Studies only included exercise as leisure time physical activity.

(20) PA was accumulated based on 20 minute bouts.

(30) PA was accumulated based on 30 minute bouts.

least 10 minutes, 14 of the 53 studies recorded all moderate or vigorous PA (see Table 2).

\section{General PA prevalence in older adults}

Tables 3 and 4 present a synopsis of the findings relating to older people's PA levels from each country. Across the 53 papers, the percentage of older adults meeting the guidelines ranged from $2.4 \%$ [55] to $83 \%$ [49] with most studies reporting that $20-60 \%$ of the samples met the guideline. When LTPA was measured, 20 studies excluded household work with reported PA prevalence ranging from $6.23 \%$ [58] to $67.51 \%$ [27], while 14 included household work with reported PA prevalence of $10.86 \%$ [31] to $66.7 \%$ [16]. Seventeen studies measured all domains of PA (including occupational, household, transportation and recreational PA) with reported PA prevalence of $11.67 \%$ [31] to $77.22 \%$ [51], and two studies reported that $31.7 \%$ [13] and $62.4 \%$ [45] of older people achieved sufficient PA through walking. In six papers PA prevalence was reported by age group, gender group, or residential area group (rural versus urban), but not for the sample overall.

\section{Self-reported PA}

In 48 subjectively measured PA studies, 29 studies adopted the more detailed PA guidelines and 21 studies adopted the less detailed PA guidelines (5 studies adopted 2 criteria). Studies that employed the more detailed guidelines reported PA prevalence ranging from $6.2 \%$ in the Health Survey for England [38] to $82.6 \%$ in an urban Chinese sample [47]. Using the less detailed guidelines (criteria), sufficient PA in the other studies increased from $21.3 \%$ [34] to $83.0 \%$ [49] in a small older female sample.

Those studies that accumulated PA data by 10 minutes bouts reported a relatively lower PA prevalence. The two studies which only included PA data of more than 30 minute sessions reported the lowest PA prevalence (10.9\% [30]; 6.23\% [58]) across all the subjectively measured PA studies.

\section{Objectively measured PA}

Six studies used accelerometers and reported extractable data (i.e. proportion of the sample meeting the criterion of sufficient PA rather than measures of central tendency). 
Table 3 Physical activity studies which used subjective measures

\begin{tabular}{|c|c|c|c|c|c|c|}
\hline NO. & $\begin{array}{l}\text { Author, year of } \\
\text { publication, country }\end{array}$ & $\begin{array}{l}\text { Data collection } \\
\text { period }\end{array}$ & Sample size $^{\Delta}$ & Data source & $\begin{array}{l}\mathrm{N} \text { and \%of sufficiently } \\
\text { active old people }\end{array}$ & Comment \\
\hline \multirow[t]{3}{*}{1} & \multirow{3}{*}{$\begin{array}{l}\text { Merom et al. [6] } \\
\text { 2006, Australia }\end{array}$} & 1989-1990 & $8160,(60+y r)$ & National health survey & $2285,28 \%$ & \multirow{3}{*}{$\begin{array}{l}\text { High quality, National representative } \\
\text { sample, Validated questionnaire }\end{array}$} \\
\hline & & 1995-1996 & $7620,(60+y r)$ & National health survey & $2027,26.6 \%$ & \\
\hline & & 2000 & 4359, $(60+y r)$ & National health survey & $1125,25.8 \%$ & \\
\hline 2 & $\begin{array}{l}\text { Jerome et al. [7] } \\
\text { 2006, USA }\end{array}$ & $1991(I)$ and 1994 (II) & $\begin{array}{l}243 F(70-79 y r) \\
\text { without functional } \\
\text { deficit }\end{array}$ & $\begin{array}{l}\text { Women's Health and } \\
\text { Aging Studies I and II }\end{array}$ & $53,22 \%$ & $\begin{array}{l}\text { High quality, Regional representative } \\
\text { sample, Validated questionnaire }\end{array}$ \\
\hline \multirow[t]{6}{*}{3} & \multirow{6}{*}{$\begin{array}{l}\text { Brownson et al. [8] } \\
\text { 2000, USA }\end{array}$} & \multirow[t]{6}{*}{1996} & \multirow{6}{*}{$\begin{array}{l}\text { 24406, } 14307(65-74 y r) \\
\text { and } 10099(75+y r)\end{array}$} & \multirow{6}{*}{$\begin{array}{l}\text { Behavioural Risk Factor } \\
\text { Surveillance System } \\
\text { (BRFSS) }\end{array}$} & CDC-ACSM 1995 & \multirow{6}{*}{$\begin{array}{l}\text { High quality National representative } \\
\text { sample Validated questionnaire }\end{array}$} \\
\hline & & & & & $5696,23.34 \%$ & \\
\hline & & & & & $65-74: 25.7 \% 75+: 20.0 \%$ & \\
\hline & & & & & SGR 1996 & \\
\hline & & & & & $6480,26.55 \%$ & \\
\hline & & & & & $65-74: 30.4 \% 75+: 21.1 \%$ & \\
\hline 4 & $\begin{array}{l}\text { Brach et al. [9] } \\
\text { 2004, USA }\end{array}$ & 1998 & 3075 (70-79), $1584 \mathrm{~F}$ & Health $A B C$ study & $\begin{array}{l}\text { 750, } 24.4 \% \text { Men: } 33.07 \% \\
\text { Women: } 16.22 \%\end{array}$ & $\begin{array}{l}\text { High quality Regional representative } \\
\text { sample Standardized questionnaire }\end{array}$ \\
\hline 5 & $\begin{array}{l}\text { Phongsavan et al. } \\
\text { [10] 2004, Australia }\end{array}$ & 1998 & $4321,1675 \mathrm{M}$ & $\begin{array}{l}\text { NSW State health } \\
\text { survey }\end{array}$ & $\begin{array}{l}\text { Domestic PA excluded 1634, } \\
\text { 37.82\% Men: } 46.7 \% \text { Women: 32.2\% } \\
\text { Domestic PA included 2184, } \\
\text { 50.55\% Men: } 59.8 \% \text { Women: } 44.7 \%\end{array}$ & $\begin{array}{l}\text { High quality Regional representative } \\
\text { sample Validated questionnaire }\end{array}$ \\
\hline \multirow[t]{15}{*}{6} & \multirow{15}{*}{$\begin{array}{l}\text { Chau et al. [11], } \\
\text { 2008, Australia }\end{array}$} & \multirow[t]{3}{*}{1998} & \multirow[t]{3}{*}{$2068,(65+y r)$} & \multirow{3}{*}{$\begin{array}{l}\text { NSW Population health } \\
\text { survey }\end{array}$} & $748,36.15 \%$ & \multirow{3}{*}{$\begin{array}{l}\text { High quality Regional representative } \\
\text { sample Validated questionnaire }\end{array}$} \\
\hline & & & & & 65-74: $1357,40.1 \%$ & \\
\hline & & & & & $75+: 711,28.6 \%$ & \\
\hline & & \multirow[t]{3}{*}{2002} & \multirow[t]{3}{*}{$3420,(65+y r)$} & \multirow{3}{*}{$\begin{array}{l}\text { NSW Population health } \\
\text { survey }\end{array}$} & $1295,37.88 \%$ & \\
\hline & & & & & 65-74: $2038,41.8 \%$ & \\
\hline & & & & & $75+: 1382,32.1 \%$ & \\
\hline & & \multirow[t]{3}{*}{2003} & \multirow[t]{3}{*}{$3577,(65+y r)$} & \multirow{3}{*}{$\begin{array}{l}\text { NSW Population health } \\
\text { survey }\end{array}$} & $1217,34.02 \%$ & \\
\hline & & & & & 65-74: 2022,39.8\% & \\
\hline & & & & & $75-: 1555,26.5 \%$ & \\
\hline & & \multirow[t]{3}{*}{2004} & \multirow[t]{3}{*}{ 2706, (65+yr) } & \multirow{3}{*}{$\begin{array}{l}\text { NSW Population health } \\
\text { survey }\end{array}$} & $1073,39.66 \%$ & \\
\hline & & & & & $65-74: 1552,45.5 \%$ & \\
\hline & & & & & $75-: 1154,31.8 \%$ & \\
\hline & & \multirow[t]{3}{*}{2005} & \multirow[t]{3}{*}{$3391,(65+y r)$} & \multirow{3}{*}{$\begin{array}{l}\text { NSW Population health } \\
\text { survey }\end{array}$} & $1350,39.82 \%$ & \\
\hline & & & & & 65-74: $1921,45.5 \%$ & \\
\hline & & & & & $75-: 1470,32.4 \%$ & \\
\hline
\end{tabular}

High quality Regional representative sample Standardized questionnaire High quality Regional representative High quality Regional representative sample Validated questionnaire 
Table 3 Physical activity studies which used subjective measures (Continued)

\begin{tabular}{|c|c|c|c|c|c|c|}
\hline 7 & $\begin{array}{l}\text { Hamdorf et al. [12] } \\
\text { 2002, Australia }\end{array}$ & 1998 & $773,(60+y r)$ & $\begin{array}{l}\text { Social Environmental } \\
\text { Risk Context } \\
\text { Information System } \\
\text { (SERCIS) }\end{array}$ & $\begin{array}{l}\text { Didn't report the prevalence of } \\
\text { whole sample } 60-64: 181,50.8 \% \text {, } \\
85+: 26,15.4 \% \text { Figure } 2 \text { showed the } \\
\text { trend of declining }\end{array}$ & $\begin{array}{l}\text { High quality Regional representative } \\
\text { sample Validated questionnaire }\end{array}$ \\
\hline \multirow[t]{18}{*}{8} & \multirow{18}{*}{$\begin{array}{l}\text { Merom et al. [13] } \\
\text { 2009, Australia }\end{array}$} & \multirow[t]{3}{*}{1998} & \multirow[t]{3}{*}{$2068,(65+y r)$} & \multirow{3}{*}{$\begin{array}{l}\text { The NSW Population } \\
\text { health survey }\end{array}$} & $532,25.73 \%$ & \multirow{18}{*}{$\begin{array}{l}\text { High quality Regional representative } \\
\text { sample Validated questionnaire }\end{array}$} \\
\hline & & & & & 65-74: 1357,28.0 & \\
\hline & & & & & $75+: 711,21.4$ & \\
\hline & & \multirow[t]{3}{*}{2002} & \multirow[t]{3}{*}{$3420,(65+y r)$} & \multirow{3}{*}{$\begin{array}{l}\text { The NSW Population } \\
\text { health survey }\end{array}$} & $970,28.35 \%$ & \\
\hline & & & & & 65-74: 2038,30.9\% & \\
\hline & & & & & $75+: 1382,24.6 \%$ & \\
\hline & & \multirow[t]{3}{*}{2003} & \multirow[t]{3}{*}{$3577,(65+y r)$} & \multirow{3}{*}{$\begin{array}{l}\text { The NSW Population } \\
\text { health survey }\end{array}$} & $899,25.13 \%$ & \\
\hline & & & & & 65-74: 2022,29.3\% & \\
\hline & & & & & $75+: 1555,19.7 \%$ & \\
\hline & & \multirow[t]{3}{*}{2004} & \multirow[t]{3}{*}{$2706,(65+y r)$} & \multirow{3}{*}{$\begin{array}{l}\text { The NSW Population } \\
\text { health survey }\end{array}$} & $781,28.85 \%$ & \\
\hline & & & & & 65-74:1552, 32.3\% & \\
\hline & & & & & $75+: 1154,24.2 \%$ & \\
\hline & & \multirow[t]{3}{*}{2005} & \multirow[t]{3}{*}{$3391,(65+y r)$} & \multirow{3}{*}{$\begin{array}{l}\text { The NSW Population } \\
\text { health survey }\end{array}$} & $985,29.04 \%$ & \\
\hline & & & & & 65-74:1921, 32.9\% & \\
\hline & & & & & $75+: 1470,24.0 \%$ & \\
\hline & & \multirow[t]{3}{*}{2006} & \multirow[t]{3}{*}{$2388,(65+y r)$} & \multirow{3}{*}{$\begin{array}{l}\text { The NSW Population } \\
\text { health survey }\end{array}$} & $744,31.17 \%$ & \\
\hline & & & & & 65-74: 1318,35.7\% & \\
\hline & & & & & $75+: 1070,25.6 \%$ & \\
\hline 9 & $\begin{array}{l}\text { Heesch \& Brown [14] } \\
\text { 2008, Australia }\end{array}$ & 1999 & 3613 F, $75.28(73-78$ yr) & $\begin{array}{l}\text { Australian Longitudinal } \\
\text { Study on } \\
\text { Women's Health }\end{array}$ & $1572,43.5 \%$ & $\begin{array}{l}\text { High quality National representative } \\
\text { sample Validated questionnaire }\end{array}$ \\
\hline 10 & $\begin{array}{l}\text { Lim \& Taylor [15] 2005, } \\
\text { Australia }\end{array}$ & 1999 & $\begin{array}{l}8881,5045 \text { F, } 73.8 \\
(95 \% C l 73.6-74.0 y r)\end{array}$ & $\begin{array}{l}\text { NSW Older People's } \\
\text { Health } \\
\text { Survey (OPHS) }\end{array}$ & $\begin{array}{l}4343,48.9 \% \text { Women, } 39.7 \% \text {, } \\
\text { Men, } 61.1 \%\end{array}$ & $\begin{array}{l}\text { High quality Regional representative } \\
\text { sample Validated questionnaire }\end{array}$ \\
\hline 11 & $\begin{array}{l}\text { Lawlor et al. [16] } \\
\text { 2002, UK }\end{array}$ & 1999-2000 & 2341 F (60-79yr) & $\begin{array}{l}\text { British Women's Heart } \\
\text { and } \\
\text { Health Study }\end{array}$ & $\begin{array}{l}\text { Domestic PA included 1561, 66.7\% } \\
\text { Domestic PA excluded 492, } 21 \% \\
\text { Domestic activity (heavy house } \\
\text { work and heavy gardening/DIY) }\end{array}$ & $\begin{array}{l}\text { High quality National representative } \\
\text { sample Validated questionnaire }\end{array}$ \\
\hline 12 & $\begin{array}{l}\text { Hillsdon et al. [17] } \\
\text { 2008, UK }\end{array}$ & 1999-2001 & 4103 F, (60-79yr) & $\begin{array}{l}\text { British Women's Heart } \\
\text { and } \\
\text { Health Study }\end{array}$ & $926,22.57 \%$ & $\begin{array}{l}\text { High quality National representative } \\
\text { sample Validated questionnaire }\end{array}$ \\
\hline
\end{tabular}


Table 3 Physical activity studies which used subjective measures (Continued)

\begin{tabular}{|c|c|c|c|c|c|c|c|c|}
\hline 13 & $\begin{array}{l}\text { Brown et al. [18] } \\
\text { 2003, USA }\end{array}$ & 2001 & $30146(65+y r), 17081 \mathrm{~F}$ & BRFSS & \multicolumn{2}{|c|}{$\begin{array}{l}\text { 11305, 37.5\%, Men: } \\
42.03 \%\end{array}$} & Women: $31.90 \%$ & $\begin{array}{l}\text { High quality National representative } \\
\text { sample, Validated questionnaire }\end{array}$ \\
\hline 14 & $\begin{array}{l}\text { Brown et al. [19] } \\
\text { 2005, USA }\end{array}$ & 2001 & $22174(65+y r), 13834 \mathrm{~F}$ & BRFSS & \multicolumn{2}{|c|}{$\begin{array}{l}\text { 9202, 41.50\%, Men: } \\
\text { 47.38\%, 65-74: } \\
5369,49.3 \% 75+: \\
2971,43.9 \%\end{array}$} & $\begin{array}{l}\text { Women: } 37.96 \% \\
65-74: 8059 \\
41.3 \% \text {, } 75+: 5775,33.3 \%\end{array}$ & $\begin{array}{l}\text { High quality National representative } \\
\text { sample, Validated questionnaire }\end{array}$ \\
\hline \multirow[t]{10}{*}{15} & \multirow{10}{*}{$\begin{array}{l}\text { Ashe et al. [20] } \\
\text { 2009, Canada }\end{array}$} & \multirow[t]{10}{*}{$2000-2001$} & \multirow{10}{*}{$\begin{array}{l}24233(65+y r), 60 \% F \\
(76 \% \text { reported having } \\
\text { one or more chronic } \\
\text { diseases) }\end{array}$} & \multirow{10}{*}{$\begin{array}{l}\text { Canadian Community } \\
\text { Health Survey Cycle } 1.1\end{array}$} & \multicolumn{2}{|c|}{ No chronic disease } & One or more chronic disease & \multirow{10}{*}{$\begin{array}{l}\text { High quality National representative } \\
\text { sample Validated questionnaire }\end{array}$} \\
\hline & & & & & Total & $7318,30.2 \%$ & $5622,23.2 \%$ & \\
\hline & & & & & \multicolumn{3}{|c|}{ Women } & \\
\hline & & & & & $65-74$ & $26.7 \%$ & $21.9 \%$ & \\
\hline & & & & & $75-84$ & $18.1 \%$ & $12.7 \%$ & \\
\hline & & & & & $85+$ & $14.5 \%$ & $7.4 \%$ & \\
\hline & & & & & \multicolumn{3}{|l|}{ Men } & \\
\hline & & & & & $65-74$ & $40.6 \%$ & $38.0 \%$ & \\
\hline & & & & & $75-84$ & $35.9 \%$ & $27.3 \%$ & \\
\hline & & & & & $85+$ & $26.9 \%$ & $20.8 \%$ & \\
\hline \multirow[t]{2}{*}{16} & \multirow[t]{2}{*}{$\begin{array}{l}\text { US CDC [21] } \\
2007, \text { USA }\end{array}$} & 2001 & $\begin{array}{l}(65+y r),(\text { whole sample } \\
205,140)\end{array}$ & BRFSS & \multicolumn{3}{|c|}{ Men $43.1 \%$ women $32.2 \%$} & $\begin{array}{l}\text { High quality National representative } \\
\text { sample Validated questionnaire }\end{array}$ \\
\hline & & 2005 & $\begin{array}{l}(65+y r),(\text { whole sample } \\
356,112)\end{array}$ & BRFSS & \multicolumn{3}{|c|}{ Men $44.5 \%$ women $36.3 \%$} & \\
\hline \multirow[t]{3}{*}{17} & \multirow{3}{*}{$\begin{array}{l}\text { Muntner et al [22] } \\
\text { 2005, China }\end{array}$} & \multirow[t]{3}{*}{$2000-2001$} & \multirow[t]{3}{*}{1824 (65-79yr), $939 \mathrm{M}$} & \multirow[t]{3}{*}{ InterASIA study } & \multicolumn{3}{|c|}{$821,45 \%$} & \multirow{3}{*}{$\begin{array}{l}\text { High quality National representative } \\
\text { sample Validated questionnaire }\end{array}$} \\
\hline & & & & & \multicolumn{3}{|c|}{ Rural $52.7 \%$} & \\
\hline & & & & & \multicolumn{3}{|c|}{ Urban $9.8 \%$} & \\
\hline 18 & $\begin{array}{l}\text { Benedetti et al. [23] } \\
\text { 2008, Brazil }\end{array}$ & 2002 & $\begin{array}{l}875,71.6 \pm 7.9 \\
(65-101 \mathrm{yr}), 437 \mathrm{M}\end{array}$ & $\begin{array}{l}\text { A representative survey: } \\
\text { Profile } \\
\text { of Old Persons in the } \\
\text { Municipality } \\
\text { of Florianópolis }\end{array}$ & \multicolumn{2}{|c|}{ 519, 59.3\% Men 63.6\% } & Women 55\% & $\begin{array}{l}\text { High quality Regional representative } \\
\text { sample Validated questionnaire }\end{array}$ \\
\hline 19 & $\begin{array}{l}\text { Ding et al. [24] } \\
\text { 2009, China }\end{array}$ & 2002 & $799(60+y r)$ & $\begin{array}{l}2002 \text { Beijing Nutrition } \\
\text { and } \\
\text { Health Survey }\end{array}$ & \multicolumn{2}{|c|}{$\begin{array}{l}254,31.79 \% 60-74: 673 \\
33 \% 75+: 126,25.3 \%\end{array}$} & & $\begin{array}{l}\text { High quality Regional representative } \\
\text { sample Validated questionnaire }\end{array}$ \\
\hline 20 & $\begin{array}{l}\text { Hallal et al. [25] } \\
\text { 2003, Brazil }\end{array}$ & 2002 & $583(60+y r), 360 F$ & $\begin{array}{l}\text { Cross-sectional survey } \\
\text { Pelotas, Brazil }\end{array}$ & \multicolumn{2}{|c|}{$\begin{array}{l}270,46.3 \% 60- \\
69: 307,56.2 \% 70 \\
+: 276,35.3 \% \text { Men } 60- \\
69: 124,55.4 \% 70 \\
+: 99,43 \%\end{array}$} & $\begin{array}{l}\text { Women 60-69:183, } \\
56.8 \% \text { 70+: } 177,30.3 \%\end{array}$ & $\begin{array}{l}\text { High quality Regional representative } \\
\text { sample Validated questionnaire }\end{array}$ \\
\hline 21 & & 2007 & & & \multicolumn{3}{|c|}{ 60-69: 42.7\% 70+: 23.7\% } & \\
\hline
\end{tabular}


Table 3 Physical activity studies which used subjective measures (Continued)

\begin{tabular}{|c|c|c|c|c|c|c|c|}
\hline & $\begin{array}{l}\text { Knuth et al. [26] } \\
2010 \text { Brazil }\end{array}$ & & $\begin{array}{l}\text { (65+yr), (whole sample } \\
2969)\end{array}$ & $\begin{array}{l}\text { Cross-sectional survey } \\
\text { Pelotas, Brazil }\end{array}$ & & & $\begin{array}{l}\text { High quality Regional representative } \\
\text { sample Validated questionnaire }\end{array}$ \\
\hline \multirow[t]{11}{*}{22} & \multirow{11}{*}{$\begin{array}{l}\text { Meyer [27] } \\
2005 \text { Switzerland }\end{array}$} & \multirow[t]{11}{*}{2002} & \multirow[t]{11}{*}{$4057(65+y r)$} & \multirow[t]{11}{*}{ Swiss Health Survey* } & \multirow{2}{*}{\multicolumn{2}{|c|}{$\begin{array}{l}\text { Total: } 2739,67.51 \% \\
65-79: 3257,80.9 \%\end{array}$}} & \multirow{11}{*}{$\begin{array}{l}\text { High quality National representative } \\
\text { sample Validated questionnaire }\end{array}$} \\
\hline & & & & & & & \\
\hline & & & & & \multicolumn{2}{|l|}{$80+: 800,58.1 \%$} & \\
\hline & & & & & \multicolumn{2}{|l|}{ Sports/exercise } & \\
\hline & & & & & \multicolumn{2}{|l|}{ Total: 515, 12.7\% } & \\
\hline & & & & & \multicolumn{2}{|l|}{ 65-79: $25.5 \%$} & \\
\hline & & & & & \multicolumn{2}{|l|}{$80+: 24.77 \%$} & \\
\hline & & & & & \multicolumn{2}{|l|}{ Habitual } & \\
\hline & & & & & \multicolumn{2}{|c|}{ Total: 2314, 57.04\% } & \\
\hline & & & & & \multicolumn{2}{|l|}{$80+: 51.7 \%$} & \\
\hline & & & & & \multicolumn{2}{|l|}{ 65-79: $45.4 \%$} & \\
\hline 23 & $\begin{array}{l}\text { Frank et al. [28] } \\
2010 \text {, USA }\end{array}$ & $2001-2002$ & $1970(65+y r)$ & $\begin{array}{l}\text { Strategies for } \\
\text { Metropolitan } \\
\text { Atlanta's Regional } \\
\text { Transportation } \\
\text { and Air Quality } \\
\text { (SMARTRAQ study, } \\
\text { Atlanta region) }\end{array}$ & \multicolumn{2}{|c|}{$\begin{array}{l}\text { Total: } 791,40.16 \% \text { 65-74:1198, } \\
42.7 \% \text { 75-84: } 622,38.0 \% \\
85+: 150,28.8 \%\end{array}$} & $\begin{array}{l}\text { High quality Regional representative } \\
\text { sample Validated questionnaire }\end{array}$ \\
\hline 24 & $\begin{array}{l}\text { Guinn et al. [29] } \\
\text { 2002, USA }\end{array}$ & $\begin{array}{l}\text { Unclear,earlier than } \\
2002\end{array}$ & $\begin{array}{l}244(60-81 y r) \\
162 \text { or } 170 \mathrm{~F}\end{array}$ & $\begin{array}{l}\text { Cross-sectional survey } \\
\text { in a } \\
\text { retirement area, Texas }\end{array}$ & \multicolumn{2}{|c|}{$\begin{array}{l}\text { 136, 55.74\% men: (82) } 74, \\
66.57 \% \text { women: (162)170, 50.59\% }\end{array}$} & $\begin{array}{l}\text { Weak quality Sample bias likely due } \\
\text { to } \\
\text { recruitment procedure Testing of } \\
\text { instrument not reported. \% of male } \\
\text { or female was inconsistent in the } \\
\text { paper }\end{array}$ \\
\hline \multirow[t]{2}{*}{25} & \multirow[t]{2}{*}{$\begin{array}{l}\text { Taylor-Piliae et al. } \\
\text { [30] 2006, USA }\end{array}$} & \multirow[t]{2}{*}{ 2001-2004 } & \multirow[t]{2}{*}{$\begin{array}{l}1010,65.8 \pm 2.8 \\
(60-69 y r), 631 \mathrm{M}\end{array}$} & \multirow{2}{*}{$\begin{array}{l}\text { Healthy control of } \\
\text { Atherosclerotic } \\
\text { disease vascular } \\
\text { function and } \\
\text { genetic epidemiology } \\
\text { (ADVANCE) Study }\end{array}$} & $\begin{array}{l}\text { All domain PA n } \\
\text { Stanford Seven-1 } \\
646,64 \%\end{array}$ & Recall & \multirow{2}{*}{$\begin{array}{l}\text { High quality Sample was selected } \\
\text { from } \\
\text { a medical insurance programme } \\
\text { Validated questionnaire }\end{array}$} \\
\hline & & & & & \multicolumn{2}{|c|}{$\begin{array}{l}\text { On-the-job activity and leisure-time } \\
\text { activities measured by Stanford } \\
\text { Brief Activity Survey } 616,61 \% \text {, } \\
\text { Men } 61.5 \% \text { women } 60.7 \%\end{array}$} & \\
\hline \multirow[t]{4}{*}{26} & \multirow[t]{4}{*}{$\begin{array}{l}\text { Allender et al, [31] } \\
\text { 2008, UK }\end{array}$} & \multirow[t]{4}{*}{2003} & \multirow[t]{4}{*}{$1181(65+y r)$} & \multirow[t]{4}{*}{$\begin{array}{l}\text { Health Survey for } \\
\text { England (HsfE) }\end{array}$} & $\begin{array}{l}\text { Occupational } \\
\text { PA included }\end{array}$ & $\begin{array}{l}\text { Occupational } \\
\text { PA excluded }\end{array}$ & \multirow[t]{4}{*}{$\begin{array}{l}\text { High quality National representative } \\
\text { sample Validated questionnaire }\end{array}$} \\
\hline & & & & & $138,11.67 \%$ & $128,10.86 \%$ & \\
\hline & & & & & Men:14.68\% & Men: $13.3 \%$ & \\
\hline & & & & & 65-74: $740,18 \%$ & 65-74: 740,16.4 & \\
\hline
\end{tabular}


Table 3 Physical activity studies which used subjective measures (Continued)

\begin{tabular}{|c|c|c|c|c|c|c|c|c|}
\hline & & & & & \multicolumn{2}{|c|}{$75+: 441,9.1 \%$} & \multicolumn{2}{|l|}{$75+: 441,8.7 \%$} \\
\hline & & & & & \multicolumn{2}{|c|}{ Women: $9.41 \%$} & \multicolumn{2}{|l|}{ Women: 9.03\% } \\
\hline & & & & & \multicolumn{2}{|c|}{ 65-74: $853,13.7 \%$} & 65-74: $853,13.0 \%$ & \\
\hline & & & & & \multicolumn{2}{|c|}{$75+:$ : $717,4.3 \%$} & $75+: 717,4.3 \%$ & \\
\hline \multirow[t]{5}{*}{27} & \multirow{5}{*}{$\begin{array}{l}\text { Florindo et al. [32] } \\
\text { 2009, Brazil }\end{array}$} & \multirow[t]{5}{*}{2003} & \multirow{5}{*}{$\begin{array}{l}\text { (60-65yr), the whole } \\
\text { sample was } 1318\end{array}$} & \multirow{5}{*}{$\begin{array}{l}\text { Health Survey of the } \\
\text { Municipality } \\
\text { of Sao Paulo }\end{array}$} & \multicolumn{3}{|c|}{ Total PA 899, 68.2\% } & \multirow{5}{*}{$\begin{array}{l}\text { High quality Regional representative } \\
\text { sample Validated questionnaire }\end{array}$} \\
\hline & & & & & \multicolumn{3}{|c|}{ LTPA 232, 17.6\% } & \\
\hline & & & & & \multicolumn{3}{|c|}{ Transportation 83, 6.3\% } & \\
\hline & & & & & \multicolumn{3}{|c|}{ Occupational 250, 19\% } & \\
\hline & & & & & \multicolumn{3}{|c|}{ Household 585, 44.4\% } & \\
\hline \multirow[t]{3}{*}{28} & \multirow{3}{*}{$\begin{array}{l}\text { Joubert et al. [33] } \\
\text { 2007, South Africa }\end{array}$} & \multirow[t]{3}{*}{2003} & \multirow[t]{3}{*}{$(60+y r)$} & \multirow{3}{*}{$\begin{array}{l}\text { World Health Survey } \\
2003\end{array}$} & \multicolumn{2}{|l|}{ Men } & Women & \multirow{3}{*}{$\begin{array}{l}\text { High quality National representative } \\
\text { sample Method of sample } \\
\text { recruitment } \\
\text { not reported Validated questionnaire }\end{array}$} \\
\hline & & & & & \multicolumn{2}{|c|}{ 60-69: $21.2 \%$} & 60-69: $18.4 \%$ & \\
\hline & & & & & \multicolumn{2}{|c|}{$70+: 22.3 \%$} & $70+: 10 \%$ & \\
\hline 29 & $\begin{array}{l}\text { Meisner et al. [34] } \\
\text { 2010, Canada }\end{array}$ & 2003 & $12042(60+y r), 6823 F$ & $\begin{array}{l}\text { Part of Canadian } \\
\text { Community } \\
\text { Health Survey (CCHS; } \\
\text { Cycle 2.1) }\end{array}$ & \multicolumn{2}{|c|}{$2565,21.3 \%$} & & $\begin{array}{l}\text { High quality National representative } \\
\text { sample Validated questionnaire }\end{array}$ \\
\hline \multirow[t]{5}{*}{30} & \multirow{5}{*}{$\begin{array}{l}\text { Mummery et al. [35] } \\
\text { 2007, New Zealand }\end{array}$} & \multirow[t]{5}{*}{2003} & \multirow[t]{5}{*}{$1894(60+y r), 1009 \mathrm{~F}$} & Obstacles to Action & $974,51.49$ & & & High quality National representative \\
\hline & & & & & Men 55.2 & & Women $47.4 \%$ & sample Validated questionnaire \\
\hline & & & & & $60-64: 603$ & 56.5 & $65-69: 445,52.8 \%$ & \\
\hline & & & & & 70-74: 36 & $3,51.1 \%$ & $75-79: 270,47.7 \%$ & \\
\hline & & & & & $80+: 213$ & $32.3 \%$ & & \\
\hline 31 & McGuire et al. [36] & 2003 & $36,010 F(65+y r)$ & BFRSS & $6986,19.4$ & & & High quality National representative \\
\hline & & & & & $65-69: 100$ & $71,23.0 \%$ & 70-74: 9150, 21.3\% & sample Validated questionnaire \\
\hline & & & & & 75-79: 79 & $9,18.5 \%$ & 80-84: 5580, 15.6\% & \\
\hline & & & & & $85+: 3300$ & $13.6 \%$ & & \\
\hline 32 & $\begin{array}{l}\text { Pronk et al. [37] } \\
\text { 2004, USA }\end{array}$ & $\begin{array}{l}\text { not reported, 2002- } \\
2004\end{array}$ & $685,74.5 \pm 6.7 y r$ & $\begin{array}{l}\text { Stratified random } \\
\text { sample of } \\
\text { HealthPartners } \\
\text { membership, } \\
\text { used questions from } \\
\text { the BRFSS }\end{array}$ & $279,40.70$ & & & $\begin{array}{l}\text { High quality Stratified random } \\
\text { sample } \\
\text { Validated questionnaire }\end{array}$ \\
\hline 33 & Stamatakis et al. & 2003 & $2763(65+y r), 1187 \mathrm{M}$ & Health Survey for & Domestic & excluded & Domestic included & High quality National representative \\
\hline & & & & En & Total & $172,6.23 \%$ & $306,11.09 \%$ & sample Validated questionnaire \\
\hline & & & & & Men & $7.6 \%$ & $13.6 \%$ & \\
\hline & & & & & Women & $5.2 \%$ & $9.2 \%$ & \\
\hline
\end{tabular}


Table 3 Physical activity studies which used subjective measures (Continued)

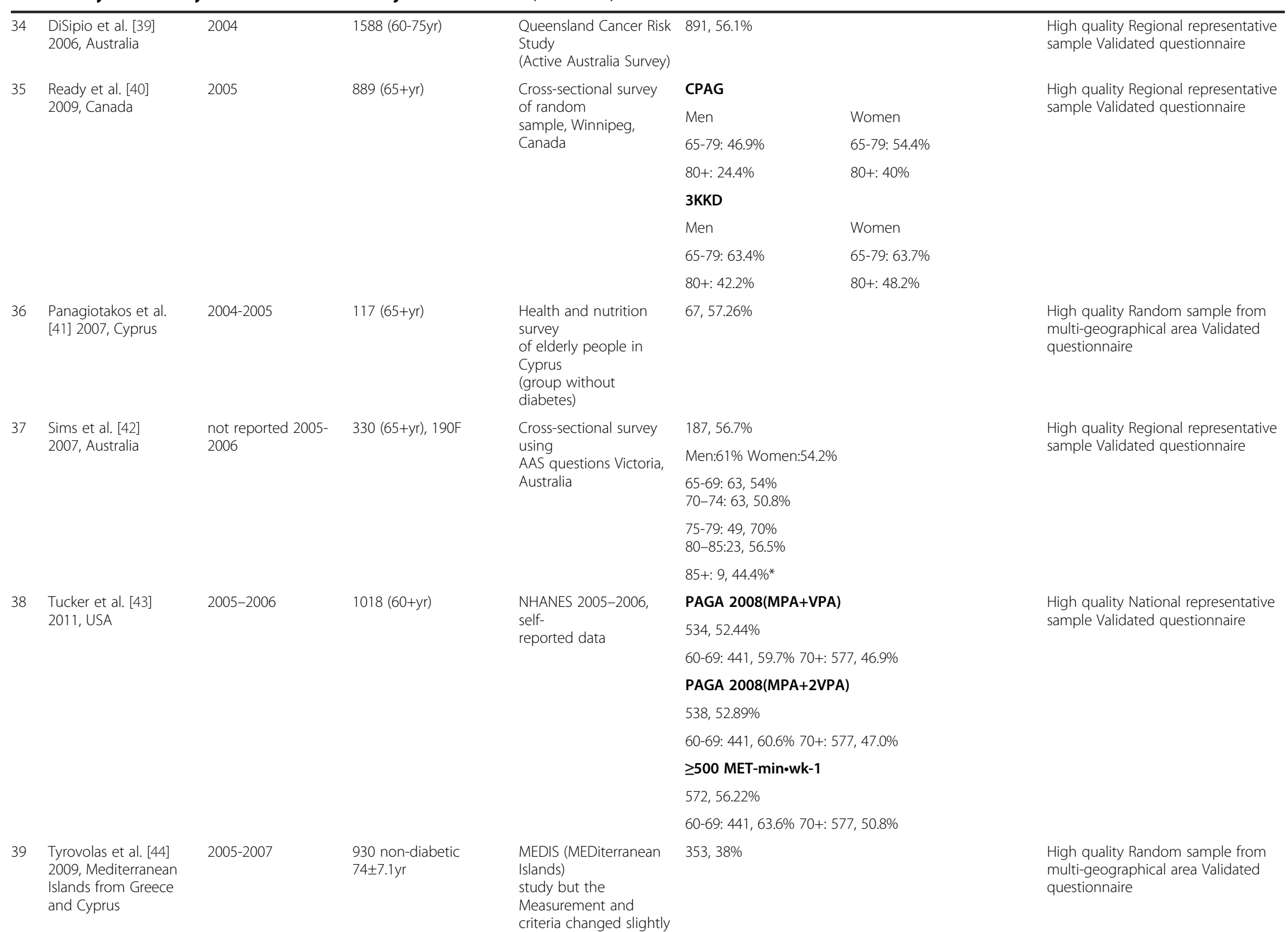
of elderly people in
Cyprus

AAS questions Victoria

75-79: $49,70 \%$

$572,56.22 \%$
60-69: 441, 59.7\% 70+: 577, 46.9\%

6-69: $441,60.6 \%$ 70+: $577,47.0 \%$

60-69: $441,63.6 \%$ 70+: $577,50.8 \%$ and Cyprus multi-geographical area Validated questionnaire 
Table 3 Physical activity studies which used subjective measures (Continued)

\begin{tabular}{|c|c|c|c|c|c|c|}
\hline & & & & $\begin{array}{l}\text { between } \\
\text { other Cyprus study }\end{array}$ & & \\
\hline 40 & $\begin{array}{l}\text { Gómez et al. [45] } \\
\text { 2010, Colombia }\end{array}$ & 2007 & $1966,70.7 \pm 7.7 y r$ & $\begin{array}{l}\text { Multilevel cross- } \\
\text { sectional study, } \\
\text { Bogotá }\end{array}$ & $1227,62.4 \%$ & $\begin{array}{l}\text { High quality Random sample from } \\
\text { multi neighbourhood in one city } \\
\text { Validate questionnaire }\end{array}$ \\
\hline 41 & $\begin{array}{l}\text { McGuire et al. [46] } \\
\text { 2010, USA }\end{array}$ & 2007 & $6138(65+y r)$ & $\begin{array}{l}\text { BRFSS } 2007 \text { of Hawaii, } \\
\text { Kansas \&Washington }\end{array}$ & $2671,43.51 \%$ & $\begin{array}{l}\text { High quality Regional representative } \\
\text { sample Validated questionnaire }\end{array}$ \\
\hline \multirow[t]{2}{*}{42} & \multirow{2}{*}{$\begin{array}{l}\text { Xu et al. [47] } \\
2009 \text {, China }\end{array}$} & \multirow[t]{2}{*}{2007} & \multirow[t]{2}{*}{$407(65-69 y r)$} & \multirow{2}{*}{$\begin{array}{l}\text { Cross- sectional survey, } \\
\text { Guangdong } \\
\text { Province using GPAQ }\end{array}$} & Rural 60.9\% & \multirow{2}{*}{$\begin{array}{l}\text { High quality Regional representative } \\
\text { sample Validated questionnaire }\end{array}$} \\
\hline & & & & & Unban 82.6\% & \\
\hline \multirow[t]{4}{*}{43} & \multirow{4}{*}{$\begin{array}{l}\text { US CDC, [48] } \\
2008, \text { USA }\end{array}$} & \multirow[t]{4}{*}{2007} & \multirow{4}{*}{$\begin{array}{l}(65+y r),(\text { whole } \\
\text { sample } 399,107)\end{array}$} & \multirow[t]{4}{*}{ BRFSS } & HP 2010 & \multirow{4}{*}{$\begin{array}{l}\text { High quality National representative } \\
\text { sample Validated questionnaire }\end{array}$} \\
\hline & & & & & $39.3 \%$ & \\
\hline & & & & & PAGA 2008 & \\
\hline & & & & & $51.2 \%$ & \\
\hline 44 & $\begin{array}{l}\text { Bird et al. [49] } \\
\text { 2008, Australia }\end{array}$ & $\begin{array}{l}\text { not reported 2006- } \\
2008\end{array}$ & $66 F(69.3 \pm 6.7 y r)$ & $\begin{array}{l}\text { Cross-sectional survey } \\
\text { Western } \\
\text { Region of Melbourne }\end{array}$ & 55, 83\%, Small sample & $\begin{array}{l}\text { Moderate quality Non-random small } \\
\text { sample Validated measurement }\end{array}$ \\
\hline \multirow[t]{4}{*}{45} & \multirow{4}{*}{$\begin{array}{l}\text { Carlson et al. [50] } \\
\text { 2010, USA }\end{array}$} & \multirow[t]{4}{*}{2008} & \multirow{4}{*}{$\begin{array}{l}2008(65+y r), \text { (whole } \\
\text { sample 21,781) }\end{array}$} & \multirow{4}{*}{$\begin{array}{l}\text { National Health } \\
\text { Interview Survey } \\
\text { employed light- } \\
\text { moderate to } \\
\text { substitute moderate }\end{array}$} & PAGA 2008 & \multirow{4}{*}{$\begin{array}{l}\text { High quality National representative } \\
\text { sample Validated questionnaire }\end{array}$} \\
\hline & & & & & $30.4 \%$ & \\
\hline & & & & & HP 2010 & \\
\hline & & & & & $22.6 \%$ & \\
\hline 46 & $\begin{array}{l}\text { Hurtig-Wennlof } \\
\text { et al. [51] 2010, } \\
\text { Sweden }\end{array}$ & $\begin{array}{l}\text { not reported before } \\
2009\end{array}$ & 54 (66-85yr), 31F & $\begin{array}{l}\text { Direct validity study } \\
\text { using } \\
\text { accelerometer- } \\
\text { measured PA }\end{array}$ & $39,72.22 \%$ & $\begin{array}{l}\text { Moderate quality Small convenience } \\
\text { sample Modified version of validated } \\
\text { instrument and Testing of } \\
\text { instrument } \\
\text { reported. }\end{array}$ \\
\hline 47 & $\begin{array}{l}\text { Shores et al. [52] } \\
\text { 2009, USA }\end{array}$ & $\begin{array}{l}\text { not reported Before } \\
2009\end{array}$ & $448(65+y r), 238 \mathrm{M}$ & $\begin{array}{l}\text { Cross-sectional survey } \\
\text { Western } \\
\text { North Carolina used 7- } \\
\text { day recall }\end{array}$ & $134,29.9 \%$ & $\begin{array}{l}\text { Moderate quality Random sample } \\
\text { Low } \\
\text { response rate Testing of instrument } \\
\text { not } \\
\text { reported. }\end{array}$ \\
\hline 48 & $\begin{array}{l}\text { Xue [53] } \\
\text { 2010, China }\end{array}$ & 2010 & 2015 (60-75yr),910M & $\begin{array}{l}\text { Coss-sectional survey } \\
\text { Nanjing, } \\
\text { Jiangsu province }\end{array}$ & $\begin{array}{l}\text { 956, 47.44\%, Men:44.2\% } \\
\text { Women: } 50.1 \%\end{array}$ & $\begin{array}{l}\text { Moderate quality Non-random } \\
\text { sample, } \\
\text { Validated questionnaire }\end{array}$ \\
\hline 49 & $\begin{array}{l}\text { Fleming et al. [54] } \\
\text { 2007, USA }\end{array}$ & 1997-2003 & $\begin{array}{l}43,259,74.9 \pm 6.9 \mathrm{yr} \\
16198 \mathrm{M}\end{array}$ & $\begin{array}{l}\text { National Health } \\
\text { Interview } \\
\text { Survey (NHIS) }\end{array}$ & $\begin{array}{l}\text { 8695, 20.1\% (age adjusted } \\
21.1 \%), \text { Men: } 25.6 \% \text {, } \\
\text { (25.8\% adjusted), Women: } \\
16.8 \%,(17.6 \% \text { adjusted) }\end{array}$ & $\begin{array}{l}\text { High quality National representative } \\
\text { sample, Validated questionnaire }\end{array}$ \\
\hline
\end{tabular}


Table 4 Physical activity studies which used objective measures

\begin{tabular}{|c|c|c|c|c|c|c|c|c|}
\hline No. & $\begin{array}{l}\text { Author, Year of } \\
\text { publication, Country }\end{array}$ & Sample size/Data source & $\begin{array}{l}\text { Data collection } \\
\text { period }\end{array}$ & Accelerometer & $\begin{array}{l}\text { Main variable } \\
\text { (cut point) }\end{array}$ & $\begin{array}{l}\text { Epoch/ } 10 \mathrm{~min} \\
\text { bouts }\end{array}$ & Guidelines & $\begin{array}{l}\text { Number and percentage } \\
\text { of sufficiently active old } \\
\text { people }\end{array}$ \\
\hline 50 & $\begin{array}{l}\text { Troiano et al. [55] } \\
\text { 2003-2004, USA }\end{array}$ & $\begin{array}{l}\text { 1260, } 624 \mathrm{M} / \text { National Health } \\
\text { and Nutritional Examination } \\
\text { Survey, NHANES }\end{array}$ & $2003-2004$ & Actigraph model 7164 & $\begin{array}{l}\text { MPA (2020-5998 } \\
\text { counts/min) VPA } \\
\text { (5999 counts/min) }\end{array}$ & $1 \mathrm{~min} / Y$ & CDC 1995 & $\begin{array}{l}30,2.4 \% \text { men } 2.5 \% \\
\text { women } 2.3 \%\end{array}$ \\
\hline \multirow[t]{2}{*}{38} & \multirow[t]{2}{*}{$\begin{array}{l}\text { Tucker et al [43] } \\
\text { 2011, USA }\end{array}$} & \multirow[t]{2}{*}{$\begin{array}{l}1018(60+y r) / N H A N E S, \\
\text { accerlerometry recorded data }\end{array}$} & \multirow[t]{2}{*}{$2005-2006$} & \multirow[t]{2}{*}{ Actigraph model 7164} & $\begin{array}{l}\text { MPA }(2020-5998 \\
\text { counts/min) VPA } \\
\text { (5999 counts/min) }\end{array}$ & $1 \mathrm{~min} / Y$ & PAGA 2008 & $\begin{array}{l}74,7.25 \% \text { 60-69: } 441 \\
8.5 \% \text { 70+: } 577,6.3 \%\end{array}$ \\
\hline & & & & & $\begin{array}{l}\text { METPA MPA (2020-5998 } \\
\text { counts/min) +VPA (5999 } \\
\text { counts/min) }\end{array}$ & $1 \min / Y$ & $\begin{array}{l}\geq 500 \mathrm{MET}^{-} \\
{\mathrm{min} \cdot \mathrm{wk}^{-1}}\end{array}$ & $\begin{array}{l}176,17.24 \% \text { 60-69: } 441 \\
26.2 \% 70+: 577,10.4 \%\end{array}$ \\
\hline 51 & $\begin{array}{l}\text { Harris et al. [56] } \\
\text { 2009, UK }\end{array}$ & $\begin{array}{l}238(65+y r) / \text { Cross-sectional } \\
\text { survey from Oxfordshire, UK }\end{array}$ & 2006 & Actigraph model GT1M & $\begin{array}{l}\text { MPA (2000-3999 } \\
\text { count/min) VPA } \\
(\geq 4000 \text { count } / \mathrm{min})\end{array}$ & $1 \mathrm{~min} / Y$ & PAGA 2008 & $6,2.5 \%$ \\
\hline 46 & $\begin{array}{l}\text { Hurtig-Wennlof } \\
\text { et al. [51] 2010, } \\
\text { Sweden }\end{array}$ & $\begin{array}{l}54(66-85 \mathrm{yr}), 23 \mathrm{M} / \text { direct } \\
\text { validity study by accelerometer }\end{array}$ & $\begin{array}{l}\text { not reported, } \\
\text { Before } 2009\end{array}$ & Actigraph, model GT1M & $\begin{array}{l}\text { MPA (mixed lifestyle } \\
\text { activities: } 760-2019 \\
\text { counts/min; ambulatory } \\
\text { activities: 2020-4944 } \\
\text { counts/min) VPA: } \\
\geq 4944 \text { counts/min }\end{array}$ & $15 S / N$ & CDC 1995 & $47,87.04 \%$ \\
\hline \multirow[t]{2}{*}{52} & \multirow[t]{2}{*}{$\begin{array}{l}\text { Colley et al. [57] } \\
\text { 2011, Canada }\end{array}$} & \multirow[t]{2}{*}{$\begin{array}{l}901 \text { (60-79yr), } 452 \text { M/Canadian } \\
\text { Health Measures Survey (CHMS) }\end{array}$} & \multirow[t]{2}{*}{ 2007-2009 } & \multirow{2}{*}{$\begin{array}{l}\text { Actical accelerometer } \\
\text { (Phillips - Respironics, } \\
\text { Oregon, USA) }\end{array}$} & \multirow{2}{*}{$\begin{array}{l}\text { MPA }(1,535-3,962 \\
\text { counts/min) VPA } \\
(\geq 3,962 \text { counts/min) }\end{array}$} & $1 \min / Y$ & CDC 1995 & $\begin{array}{l}\text { 40, } 4.5 \% \text { men: } 5.3 \% \\
\text { women: } 3.8 \%\end{array}$ \\
\hline & & & & & & $1 \min / Y$ & PAGA 2008 & $\begin{array}{l}\text { 21, } 13.1 \% \text { men: } 13.7 \% \\
\text { women: } 12.6 \%\end{array}$ \\
\hline \multirow[t]{2}{*}{53} & \multirow{2}{*}{$\begin{array}{l}\text { Davis \& Fox [3] } \\
2007 \text { UK, France } \\
\text { \& Italy }\end{array}$} & \multirow{2}{*}{$\begin{array}{l}163(76.1 \pm 4.0 \mathrm{yr}), 70 \mathrm{M} / \text { healthy } \\
\text { volunteers recruited to the Better } \\
\text { Ageing Project at four European } \\
\text { sites based in the UK, France } \\
\text { and Italy. }\end{array}$} & \multirow{2}{*}{$\begin{array}{l}\text { not reported } \\
2004-2006\end{array}$} & \multirow[t]{2}{*}{ Actigraph model 7164} & MVPA ( $\geq 1952$ counts/min) & $1 \mathrm{~min} / Y$ & CDC 1995 & 0,0 \\
\hline & & & & & MVPA ( $\geq 1952$ counts/min) & $1 \min / Y$ & PAGA 2008 & $3,1.84 \%$ \\
\hline
\end{tabular}


The actigraph accelerometer was used in five studies and Actical in one study. When measured against the less detailed guidelines, the lowest prevalence (1.84\%) was reported by Davis and Fox [3] based on an European sample and the highest was $17.2 \%$ from a US national survey, NHANES 2005-2006 [43]. Applying the more detailed guidelines, Davis and Fox [3] found nobody achieved sufficient PA and Colley [57] reported the highest proportion of $4.5 \%$ in this group with the exception of HurtigWennlof et al.'s [51] exceptional finding of 87.04\%.

However, there was a difference between the studies using self-report compared to the objective measurement of PA. In our review, two studies compared the subjective and objective data. Tucker et al.'s [43] analysis of the NHANES 2005-2006 data found that the sufficiently active group proportion defined by accelerometer measurement was $7.25 \%$ and $17.24 \%$ (using different guidelines); this increased to $54.2 \%$ when measured by questionnaire. Hurtig-Wennlof et al.'s Swedish study [51] reported a $87.04 \%$ objectively measured PA prevalence which was higher than the self-reported IPAQ data of $72.2 \%$.

\section{Gender differences in PA}

Twenty two studies reported the recommended PA prevalence in males and females separately. In general, men's PA levels were higher than women's. In the selfreported data, gender differences of PA ranged from $0.8 \%$ [30] to $21.4 \%$ [15], while the differences measured by accerlerometer were significantly lower $(0.2 \%$ and $1.5 \%)$. However, we noticed that in the self-reported total PA, increased participation in PA by women exceeded that of men in three studies $[40,53]$.

\section{Residential differences in PA}

Two Chinese papers reported the PA prevalence by place of residence and reported different results. Muntner et al. [22] reported sufficient all domain PA prevalence among $52.7 \%$ of their rural and $9.8 \%$ of their urban older people samples using the US CDC 1995 criterion from the InterASIAN Study. However, Xu et al. [47] reported that Guangdong province urban residents were more active than rural residents, with $82.6 \%$ of the urban and $60.9 \%$ of the rural samples attaining the HP 2010 goals measured by GPAQ.

\section{PA prevalence across age groups}

Eighteen studies measured PA subjectively and two studies measured PA objectively across different age groups, however, the majority divided the samples into two age groups and reported that the older old were more sedentary than the younger old. Only five studies divided the group by relatively narrow age bands. Patterns of participation in PA decreased progressively with age for both men and women although there was variation across the studies. In McGuire et al.'s study [36] PA declined from $23.0 \%$ in the $65-69$ year group to $13.6 \%$ in $85+$ year group but in Mummery et al.'s study [35], the difference between the 60-64 year group and $80+$ year group was $24.2 \%$. There was an unexpected rise in the 70-74 year age group in the Sims et al.'s [42] study although the general trends decreased with age. Although Hamdorf et al. [12] did not report the detailed percentage for each age group, we can see the same gradually declining trend from $50.8 \%$ in the $60-64$ year age group to $15.4 \%$ in $85+$ year age group in Figure 2.

\section{PA prevalence trends over time}

A crucial aspect of investigating trends over time is the comparability of methods. Differences in instruments, cut-off points, PA definitions and domains of activity investigated posed significant challenges to our review. Although nine studies reported the results of the BRFSS, not all the studies included national representative samples making it difficult to assess trends. Therefore, we only included the results of longitudinal studies or the findings from the same surveillance evaluated by the same criterion to establish the trend from seven studies. In Australia between 1990-2000 the proportion LTPA of older people aged 60 years and over meeting the PA guideline decreased from $28 \%$ to $25.8 \%$ in Merom et al.'s report [13] while the NSW Population Health Survey [11] detected a small rise in the $65+$ year age group from $36.2 \%$ in 1998 to $39.8 \%$ in 2005 with a dip to $34.0 \%$ in 2003. The trend of regular walking [13] was similar to Chau et al.'s [11] report. The USCDC compared the BRFSS data in 2001 and 2005 and reported that the US' older population was more active, with a rising prevalence of $43.1 \%$ to $44.5 \%$ in men and $32.2 \%$ to $36.3 \%$ in women [21]. Another paper using the BRFSS 2001 and BRFSS 2007 reported the same overall trend from 37.5\% to $39.3 \%$ [48]. Conversely, Knuth et al. [26] reported a clear drop in PA prevalence between 2002 and 2007 in Brazil. In 2002, the proportions of older people reaching the PAGA 2008 guideline were $56.2 \%$ and $35.3 \%$ in the 60-69 year and 70+ year age groups respectively and decreased to $42.7 \%$ and $23.7 \%$ in 2007 .

\section{Discussion}

\section{Demographic differences in PA prevalence}

One of the challenges facing the development of disease prevention programmes is the lack of reliable data for PA levels and trends [59] and the data for PA levels in older people is no exception. Generally within the included studies, the older old age groups were less likely than those of younger age to be regularly active, and women were less likely than men to achieve regular PA, especially in LTPA across both the subjectively and 


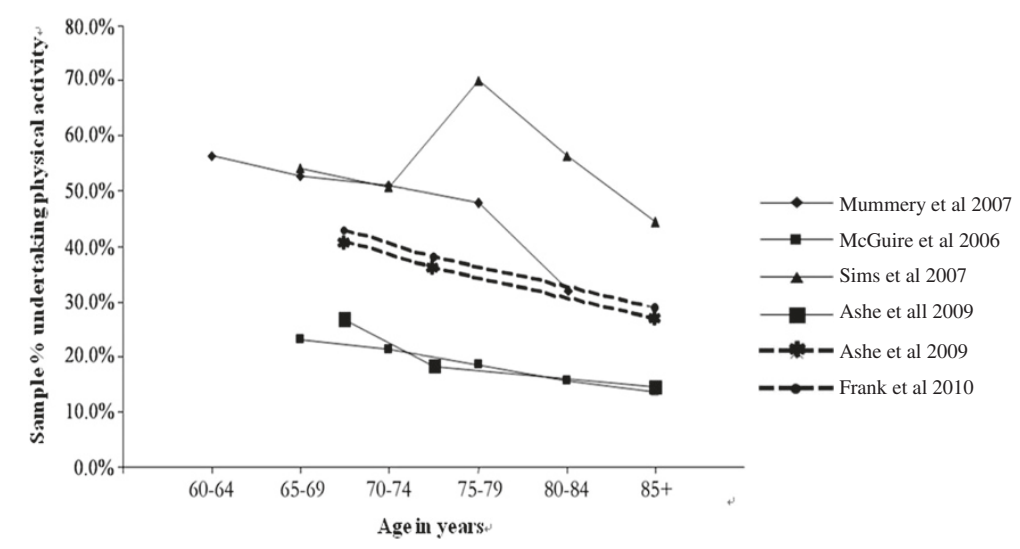

Figure 2 Physical activity prevalence across age groups.

objectively measured PA studies regardless of the PA guidelines adopted. The decline in PA with age may be the most consistent finding in PA epidemiology $[60,61]$ with the higher PA prevalence among males echoing the findings of previous studies [62] which may reflect increasing disability with age and cultural norms across the genders. However, one Australian study [42] found that the decline of LTPA across the age groups was not consistent with LTPA peaking in the 70-74 year group. Additionally three studies reported that women were more active than men in relation to total PA $[25,40,53]$. This pattern also appeared in other studies [63] which may reflect the inclusion of household activities and other non-leisure, non-sport activities [53] in the total PA, which, in many cases, are largely specific to women. However, this was not a consistent finding across the included studies regardless of the guidelines category, indicating the need to quantify household activities accurately to enable comparisons across genders.

\section{PA trends by country and over time}

In our review, there was a slightly increasing trend towards recommended PA levels in older people in Australia and the US over the last 10 years but a decrease in Brazil. Studies of time trends in PA have been conducted mostly in developed countries and their results indicate that LTPA levels appear to be increasing $[31,34,64,65]$. Nevertheless, the data indicate that substantial numbers of older adults do not engage in sufficient PA to promote their health and there is considerable variation in the levels of PA reported across countries. Further, we found little data on time trends in PA in developing countries which was echoed in a recent systematic review [66]. However, the decline in PA in Brazil [26] is perhaps indicative of changes in occupational PA with the emergence of increasing sedentary work in developing countries which will present a future public health challenge especially when combined with dietary changes. Similarly Hallal et al. have also highlighted the lower proportions of adults who are physically active in south east Asia [59].

\section{The difference between objectively and subjectively measured PA prevalence}

The only two studies comparing self-reported PA to accelerometer measured PA in our review yielded contradictory results [43,51] with Tucker et al.'s [43] findings being consistent with the majority of other similar studies [67]. It is likely that the subjective PA studies reflect social desirability, recall bias and that there is underestimation of PA using objective measurement; PA monitors are worn typically on the hip which means that they do not accurately assess upper body activities or account for movements that require extra effort, such as walking uphill or carrying loads. Hurtig-Wennlof et al.'s [51] contradictory findings were probably caused by the lower cut-off point of MPA at 760 counts per minute (contrasting with about 2000 counts/min in the other studies) and the inclusion of PA under 10 minute bouts. This highlights the need for well designed studies using objectively measured PA to generate the evidence base for public health initiatives.

\section{Review limitations}

Limitations of this review arise from the discrepancies and inconsistencies in instrumentation, PA type measured, guidelines or recommendations adopted by different researchers, algorithms, sample frames and other confounding factors. These made it difficult to make full use of the extracted data and impossible to compare the PA prevalence between different regions/countries or assess PA trends with certainty.

\section{Conclusion}

Dramatic global population ageing has brought new demands to improve older people's health by adding "quality" to their extended lives [68]. The review was undertaken 
against the background of the $\mathrm{WHO}$ recommendations on PA for adults aged 65 years and older [69]. Despite these recommendations physical inactivity is an increasing global health burden [70] with PA surveillance emerging as one of the priorities of global public health for the development of effective non-communicable disease prevention programmes [59]. International efforts to increase PA have been reported within the adult or youth populations [38,66,71-73]. However, PA levels of older adults have attracted less interest so there are limited data regarding the prevalence of various types of PA in older adults and the proportion of older people whose PA meets PA guidelines. This review is the first of its kind and revealed many methodological challenges to data analysis across the selected studies. Robust studies which recruit representative random samples and consistently employ validated measurement instruments are needed to enable comparisons in PA levels to be drawn over time and between countries. More evidence of PA levels amongst older people is needed to inform public health strategies which could extend the health and quality of life of people into old age.

\begin{abstract}
Abbreviations
AAS: Active Australia Survey; BRFSS: Behavioural Risk Factor Surveillance System; CDC-ACSM: Centers for Disease Control and Prevention and the American College of Sports Medicine; CHMS: Canadian Health Measures Survey; CCHS: Canadian Community Health Survey; GPAQ: Global Physical Activity Questionnaire; IPAQ: International Physical Activity Questionnaire; LTPA: Leisure-time physical activity; MET: Total metabolic equivalents; MPA: Moderate physical activity; NHANES: National Health and Nutritional Examination Survey; NHIS: National Health Interview Survey; PA: Physical activity; PAGA: Physical Activity Guidelines for Americans; SGR: US Surgeon General's Report; VPA: Vigorous physical activity.
\end{abstract}

\section{Competing interests}

None declared by the authors. This review was unfunded.

\section{Authors' contributions}

All authors devised the research strategy; initial screening was undertaken by FS and then checked by AEW. FS extracted the data which was checked by AEW. Disagreements were resolved by reference to IJN. All authors contributed to the data synthesis and production of the paper. All authors read and approved the final manuscript.

\section{Authors' information}

FS is a doctoral student. AEW and IJN are established health service researchers. This review was conducted to understand the prevalence and measurement of physical activity in older people.

\section{Author details}

${ }^{1}$ Second Military Medical University, School of Nursing, 800 Xiangyin Road, Shanghai 200433, PR China. ${ }^{2}$ King's College London, Florence Nightingale School of Nursing \& Midwifery, 57 Waterloo Road, London SE1 8WA, UK.

Received: 28 March 2012 Accepted: 26 April 2013

Published: 6 May 2013

\section{References}

1. Division of Ageing and Seniors: Physical activity and older adults from Canada; 2011. Available at: [http://www.phac-aspc.gc.ca/seniors-aines/indexeng.php] Accessed Aug 1, 2011.

2. Wen CP, Wai JP, Tsai MK, Yang YC, Cheng TY, Lee MC, Chan HT, Tsao CK, Tsai SP, Wu X: Minimum amount of physical activity for reduced mortality and extended life expectancy: a prospective cohort study. Lancet 2011, 378:1244-1253.
3. Davis MG, Fox KR: Physical activity patterns assessed by accelerometry in older people. Eur J Appl Physiol 2007, 100:581-589.

4. Chodzko-zajko WJ, Proctor DN, Fiatarone Singh MA, Minson CT, Nigg CR, Salem GJ, Sinner JS: American College of Sports Medicine position stand. Exercise and physical activity for older adults. Med Sci Sports Exer 2009, 41:1510-1530

5. U.S. Department of Health and Human Services: Physical Activity Guidelines for Americans; 2008. Available at: [www.health.gov/paguidelines/] Accessed Sep 11, 2011.

6. Merom D, Phongsavan P, Chey T, Bauman A: Australian Bureau of Statistics.Long-term changes in leisure time walking, moderate and vigorous exercise: Were they influenced by the national physical activity guidelines. J Sci Med Sport 2006, 9:199-208.

7. Jerome GJ, Glass TA, Mielke M, Xue QL, Andersen RE, Fried LP: Physical activity participation by presence and type of functional deficits inolder women: the Women's Health and Ageing Studies. J Gerontol A Biol Sci Med Sci 2006, 61A:1171-6.

8. Brownson RC, Jones DA, Pratt M, Blanton C, Heath GW: Measuring physical activity with the behavioral risk factor surveillance system. Med Sci Sports Exerc 2000, 32(11):1913-8.

9. Brach JS, Simonsick EM, Kritchevsky S, Yaffe K, Newman AB: Health gency and Body Composition Study Research Group. The association between physical function and lifestyle activity and exercise in the health, ageing and body composition study. J Am Geriatr Soc 2004, 52:502-509.

10. Phongsavan P, Merom D, Marshall A, Bauman A: Estimating physical activity level: the role of domestic activities. J Epidemiol Community Health 2004, 58:466-467.

11. Chau J, Smith BJ, Bauman A, Merom D, Eyeson-Annan M, Chey T, Farrell L: Recent trends in physical activity in New South Wales. Is the tide of inactivity turning? Aust New Zealand Health 2008, 32:82-85.

12. Hammdorf $P$, Starr $G$, Williams MA: A survey of physical-activity levels and functional capacity in older adults in South Australia. J Aging Phys Act 2002, 10:281-289.

13. Merom D, Chey T, Chau J, Smith BJ, Barr M, Bauman AE: Are messages about lifestyle walking being heard? Trends in walking for all purposes in New South Wales (NSW), Australia. Prev Med 2009, 48:341-344.

14. Heesch KC, Brown WJ: Do walking and leisure-time physical activity protect against arthritis in older women. $J$ Epidemiol Community Health 2008, 62:1086-1091.

15. Lim K, Taylor L: Factors associated with physical activity among older people - a population-based study. Prev Med 2005, 40:33-40.

16. Lawlor DA, Taylor M, Bedford C, Ebrahim S: Is housework good for health? Levels of physical activity and factors associated with activity in elderly women. Results from the British Women's Heart and Health Study. J Epidemiol Community Health 2002, 56:473-78.

17. Hillsdon M, Lawlor DA, Ebrahim S, Morris JN: Physical activity in older women: Associations with area deprivation and with socioeconomic position over the life course: Observations in the British Women's Heart and Health Study. J Epidemiol Community Health 2008, 62:344-350.

18. Brown DW, Balluz LS, Heath GW, Moriarty DG, Ford ES, Giles WH, Mokdad AH: Associations between recommended levels of physical activity and health-related quality of life. Findings from the 2001 Behavioral Risk Factor Surveillance System (BRFSS) survey. Prev Med 2003, 37:520-528.

19. Brown DR, Yore MM, Ham SA, Macera CA: Physical activity among adults $\geq 50$ yr with and without disabilities, BRFSS 2001. Med Sci Sports Exerc 2005, 37:620-629.

20. Ashe MC, Miller WC, Eng JJ, Noreau L: Older adults, chronic disease and leisure-time physical activity. Gerontology 2009, 55:64-72.

21. CDC, USA: Prevalence of regular physical activity among adults - United States, 2001 and 2005. Morb Mortal Wkly Rep 2007, 56:1209-1212.

22. Muntner P, Gu D, Wildman RP, Chen J, Qan W, Whelton PK, He J: Prevalence of physical activity among Chinese adults: results from the International Collaborative Study of Cardiovascular Disease in Asia. Am J Public Health 2005, 95:1631-1636.

23. Benedetti T, Borges L, Petroski E, Gonçalves LH: Physical activity and mental health status among elderly people. Rev Saude Publica 2008, 42:302-307.

24. Ding CC, Chen XR, Zhang Z, Li KJ: Exercise and walking intensity and level of Beijing adult residents. Chi J Sports Med 2009, 28:362-364. 
25. Hallal PC, Victora CG, Wells JC, Lima RC: Physical Inactivity: prevalence and associated variables in Brazilian adults. Med Sci Sports Exerc 2003, 35:1894-1900

26. Knuth $A G$, Bacchieri G, Victora CG, Hallal PC: Changes in physical activity among Brazilian adults over a 5-year period. J Epidemiol Community Health 2010, 64:591-595.

27. Meyer K, Rezny L, Breuer C, Lamprecht M, Stamm HP: Physical activity of adults aged 50 years and older in Switzerland. Soz Praventivmed 2005, 50:218-229.

28. Frank L, Kerr J, Rosenberg D, King A: Healthy ageing and where you live: Community design relationships with physical activity and body weight in older Americans. J Phys Act Health 2010, 7(1):S82-S90.

29. Guinn B, Vincent $V$ : Select physical activity determinants in independentliving elderly. Activ. Adapt. Aging 2002, 26:17-26.

30. Taylor-Piliae RE, Norton LC, Haskell WL, Mahbouda MH, Fair JM, Iribarren C, Hlatky MA, Go AS, Fortmann SP: Validation of a new brief physical activity survey among men and women aged 60-69 years. Am J Epidemiol 2006, 164(6):598-606.

31. Allender S, Foster C, Boxer A: Occupational and non-occupational physical activity and the social determinants of physical activity: results from the Health Survey for England. J Phys Act Health 2008, 5:104-16.

32. Florindo AA, Guimães W, Cesar CL, Barros MB, Alves MC, Goldblaum M: Epidemiology of leisure, transportation, occupational, and household physical activity: prevalence and associated factors. J Phys Act Health 2009, 6:625-632

33. Joubert J, Norman R, Lambert EV, Groenewald P, Schneider M, Bwi F, Bradshaw D, South African Comparative Risk Assessment Collaborating Group: Estimating the burden of disease attributable to physical inactivity in South Africa in 2000. SA Med J 2007, 97(8):725-731.

34. Meisner BA, Dogra S, Logan AJ, Baker J, Weir PL: Do or decline? Comparing the effects of physical inactivity on biopsychosocial components of successful ageing. J Health Psychol 2010, 15:688-696.

35. Mummery WK, Kolt G, Schofield G, McLean F: Associations between physical activity and other lifestyle behaviors in older New Zealanders. $J$ Phys Act Health 2007, 4:411-422

36. McGuire LC, Ahluwalia IB, Strine TW: Report from the CDC. Chronic disease-related behaviors in U.S. older women: Behavioral Risk Factor Surveillance System, 2003. J Womens Health (Larchmt) 2006, 15:3-7.

37. Pronk NP, Anderson LH, Crain AL, Martinson BC, O'Connor PJ, Sherwood NE, Whitebird RR: Meeting recommendations for multiple healthy lifestyle factors: Prevalence, clustering, and predictors among adolescent, adult, and senior health plan members. Am J Prev Med 2004, 27(2):25-33.

38. Stamatakis $E$, Ekelund $U$, Wareham N: Temporal trends in physical activity in England: The Health Survey for England 1991 to 2004. Prev Med 2007 45:416-423.

39. Disipio T, Rogers C, Newman B, Whiteman D, Eakin E, Fritschi L, Aitken J: The Queensland Cancer Risk Study: behavioural risk factor results. Aust $N$ Z J Public Health 2006, 30:375-382.

40. Ready AE, Butcher JE, Dear JB, Fieldhouse P, Harlos S, Katz A, Moffatt M, Rodriguez M, Schmalenberg J, Gardiner PF: Canada's physical activity guide recommendations are a low benchmark for Manitoba adults. Appl Physiol Nutr Metab 2009, 34:172-181.

41. Panagiotakos DB, Polystipioti A, Polychronopoulos E: Prevalence of type 2 diabetes and physical activity status in elderly men and women from Cyprus (the MEDIS Study). Asia Pac J Public Health 2007, 19:22-28.

42. Sims J, Hill K, Davidson S, Gunn J, Huang N: A snapshot of the prevalence of physical activity amongst older, community dwelling people in Victoria, Australia: patterns across the 'young-old' and 'old-old'. BMC Geriatr 2007, 7:4

43. Tucker JM, Welk GJ, Beyler NK: Adults: compliance with the physical activity guidelines for Americans. Am J Prev Med 2011, 40:454-461.

44. Tyrovolas S, Zeimbekis A, Bountziouka V, Voutsa K, Pounis G, Papoutson S, Matallinos G, Ladsukaki E, Polychronopoulos E, Lionis C, Panagiotakos DB: Factors associated with the prevalence of diabetes mellitus among elderly men and women living in Mediterranean Islands: The MEDIS study. Rev Diabet Stud 2009, 6:54-63.

45. Gómez LF, Parra DC, Buchner D, Sarmiento OL, Pinzón JD, Ardila M, Moreno J, Serrato M, Lobelo F: Built environment attributes and walking patterns among the elderly population in Bogotá. Am J Prev Med 2010, 38:592-599.

46. McGuire L, Bouldin EL, Andresen EM, Anderson LA: Examining modifiable health behaviours, body weight, and use of preventive health services among caregivers and non-caregivers age 65 years and older in Hawaii, Kansas and Washington using 2007 BRFSS. J Health Aging 2010, 14(5):373-379.

47. Xu YJ, Mao WJ, Xu XJ, Cai QM, Song XL, Nie SP, Xu HF, Xia L: Patterns and influencing factors of physical activity among residents in guangdong province. South China. J Prev Med 2009, 35:5.

48. CDC USA: Prevalence of self-reported physically active adults--United States, 2007. MMWR Morb Mortal Wkly Rep 2008, 57:1297-300.

49. Bird S, Kurowski W, Feldman S, Browning C, Lau R, Radermacher $H$, Thomas $\mathrm{S}$, Sims J: The influence of the built environment and other factors on the physical activity of older women from different ethnic communities. J Women Ageing 2009, 21:33-47.

50. Carlson SA, Fulton JE, Schoenborn CA, Loustalot F: Trend and prevalence estimates based on the 2008 Physical Activity Guidelines for Americans. Am J Prev Med 2010, 39:305-313.

51. Hurtig-Wennlof A, Hagstromer M, Olsson LA: The International Physical Activity Questionnaire modified for the elderly: aspects of validity and feasibility. Public Health Nutr 2010, 13:1847-1854.

52. Shores KA, West ST, Theriault DS, Davison EA: Extra-individual correlates of physical activity attainment in rural older adults. J Rural Health 2009, 25:8.

53. Xue MY: Effects of daily physical activities on physical health and quality of life in elderly community residents in Jiangsu Province. J Clin Rehabilitative Tissue Eng Res 2010, 14:6.

54. Fleming LE, Lee DJ, Martinez AJC, Leblanc WG, McCollister KE, Bridges KC, Christ SL, Arheart KL, Pitman T: The health behaviors of the older US worker. Am J Ind Med 2007, 50:427-437.

55. Troiano RP, Berrigan D, Dodd KW, Masse LC, Tilert T, McDowell M: Physical activity in the United States measured by accelerometer. Med Sci Sports Exerc 2007, 40:181-188.

56. Harris TJ, Owen CG, Victor CR, Adams R, Crook DG: What factors are associated with physical activity in older people, assessed objectively by accelerometry? Br J Sports Med 2009, 43:442-450.

57. Colley R, Garriguet D, Janssen I, Craig CL, Clarke J, Tremblay MS: Physical activity of Canadian children and youth: accelerometer results from the 2007 to 2009 Canadian Health Measures Survey. Health Rep 2011, 22:1-9.

58. Stamatakis $E$, Hillsdon M, Primatesta M: Domestic physical activity in relationship to multiple CVD risk factors. Am J Prev Med 2007, 32:320-327.

59. Hallal PC, Andersen LB, Bull FC, Guthold R, Haskell W, Ekelund U: Global physical activity levels: surveillance progress, pitfalls, and prospects. Lancet 2012, 380:247-257.

60. Craig CL, Russel CJ, Cameron C, Bauman A: Twenty-year trends in physical activity among Canadian adults. C J Public Health 2004, 95(1):59-63.

61. Caspersen CJ, Pereira MA, Curran KM: Changes in physical activity patterns in the United States, by sex and cross-sectional age. Med Sci Sports Exerc 2000, 32:1601-1609.

62. Curtis J, White P, McPherson B: Age and physical activity among Canadian women and men: findings from longitudinal national survey data. $J$ Ageing Phys Act 2000, 8:1-19.

63. Weller I, Corey P: The impact of excluding non-leisure energy expenditure on the relation between physical activity and mortality in women. Epidemiology 1998, 9:632-635.

64. Helkapori S, Uutela A, Prattala R: Health Behaviour and Health among FinnishPopulation, Spring 2000. Helsinki, Finland: National Public Health Institute; 2000

65. Sport and Recreation New Zealand: Results from the New Zealand Sport and PhysicalActivity Surveys (1997-2001); 2001. Available at: [http://www. activenzsurvey.org.nz/Results/SPARC-Trends-in-Participation-in-Sport-andActive-Leisure-1997-2001/Trends-Participation/] Accessed Sep 11, 2011.

66. Knuth AG, Hallal PC: Temporal trends in physical activity: a systematic review. J Phys Act Health 2009, 6:548-559.

67. Prince SA, Adamo KB, Hamel ME, Hardt J, Gorber SC, Tremblay M: A comparison of direct versus self-report measures for assessing physical activity in adults: a systematic review. Int J Behav Nutr Phys Act 2008, 6:5-56.

68. Bowling A: The psychometric properties of the older people's quality of life questionnaire, compared with the CASP-19 and the WHOQOL-OLD. Curr Gerontol Geriatr Res 2009. doi:10.1155/2009/298950.

69. World Health Organisation: Global Recommendations on Physical Activity for Health. Geneva: World Health Organisation; 2010.

70. Lee I-M, Shiroma EJ, Lobelo F, Puska P, Blair SN, Katzmarzk PT: Effect of physical inactivity on major non-communicable diseases worldwide: an 
analysis of burden of disease and life expectancy. Lancet 2012, 380:219-229.

71. Sisson SB, Katzmarzyk PT: International prevalence of physical activity in youth and adults. Obes Rev 2008, 9:606-614.

72. Vandelanotte C, Duncan MJ, Caperchione C, Hanley C, Mummery WK: Physical activity trends in Queensland (2002 to 2008): are women becoming more active than men? Aust N Z J Public Health 2010, 34:248-254.

73. Macera CA, Pratt M: Public health surveillance of physical activity. Res Q Exerc Sport 2000, 71(2):S97-103.

doi:10.1186/1471-2458-13-449

Cite this article as: Sun et al:: Physical activity in older people: a systematic review. BMC Public Health 2013 13:449.

\section{Submit your next manuscript to BioMed Central and take full advantage of:}

- Convenient online submission

- Thorough peer review

- No space constraints or color figure charges

- Immediate publication on acceptance

- Inclusion in PubMed, CAS, Scopus and Google Scholar

- Research which is freely available for redistribution 